\title{
Innovación en la educación superior: Un estudio sobre la percepción del estudiantado en Ecuador
}

Innovation in higher education: a study on the perception of students in Ecuador

\author{
Volumen 20, Número 2 \\ Mayo - Agosto \\ pp. 1-23
}

\section{Darwin Eugenio Rosales Nieto \\ Pasquale Cerbone}

Citar este documento según modelo APA

Rosales Nieto, Darwin Eugenio. y Cerbone, Pasquale. (2020). Innovación en la educación superior: Un estudio sobre la percepción del estudiantado en Ecuador. Revista Actualidades Investigativas en Educación, 20(2), 1-23. Doi. 10.15517/aie.v20i2.41644 


\title{
Innovación en la educación superior: Un estudio sobre la percepción del estudiantado en Ecuador \\ Innovation in higher education: a study on the perception of students in Ecuador
}

\section{Darwin Eugenio Rosales Nieto ${ }^{1}$ Pasquale Cerbone ${ }^{2}$}

\begin{abstract}
Resumen: Para las universidades, la innovación se convierte en un objetivo clave, y consustancial con su actividad de formar profesionales que aporten con soluciones a los problemas de la sociedad. Estas instituciones de educación superior invierten ingentes recursos para alcanzar sus metas en innovación; sin embargo, tales inversiones no siempre son percibidas por el estudiantado, lo cual genera sentimientos de insatisfacción y falta de compromiso. El objetivo de este artículo es conocer la percepción de quienes estudian en las universidades públicas -cofinanciadas y privadas de la provincia de Pichincha, en Ecuador-, con respecto a los recursos puestos a disposición por sus universidades, para orientar a las entidades públicas sobre las necesidades de innovación que en estas se presentan. Así, durante marzo y mayo de 2019, se aplicaron encuestas a una muestra de 768 estudiantes de pregrado de la mencionada provincia y se pudo evaluar con ello cuatro categorías de ítems, que evidenciaron las diferencias de percepción entre el estudiantado de los dos tipos considerados y procedente de diferentes tipos de universidades. Los resultados indican que quienes estudian en universidades públicas tienen una percepción diferente de quienes asisten a universidades privadas y cofinanciadas, y que los aspectos de infraestructura y son los más relevantes. En conclusión se recomienda priorizar la inversión en innovación, en los temas mencionados a partir de los tipos de facultades y universidades en donde la percepción es más negativa.
\end{abstract}

Palabras clave: innovación, educación superior, percepción estudiantil

\begin{abstract}
For higher education institutions, innovation becomes a key and consubstantial objective with its activity of training professionals, who provide solutions to society's problems. Universities invest huge resources to achieve their goals in innovation; however, these investments are not always perceived by the student body, which generates feelings of dissatisfaction and lack of commitment. The purpose of this article is to know the perception of those who study in public, cofounded and private universities, in the province of Pichincha, in Ecuador, about the resources made available by their universities, in order to guide public entities on the innovation needs that universities present. For this reason, during the period between March and May 2019, surveys were applied to a sample of 768 university bachelor students, from the province of Pichincha, to evaluate four categories of items, differentiating in the study the perception of students of social and exact sciences, and coming from different types of universities. The results indicate that those who study in public universities have a different perception of those who attend private and cofounded universities and those aspects of infrastructure and teaching related are the most relevant, so it is concluded that it is recommendable to prioritize investment in innovation in the mentioned topics, starting from the type of faculties and universities where the perception is more negative.
\end{abstract}

Key words: innovation; higher education; student perception

\footnotetext{
${ }^{1}$ Docente investigador de la Universidad UTE, en la Carrera de Ciencias Administrativas, Ecuador. Dirección electrónica: darwin.rosales@ute.edu.ec Orcid https://orcid.org/0000$\underline{0002-1788-782 X}$

2 Docente investigador de la Universidad UTE, en la Carrera de Ciencias Administrativas, Ecuador. Dirección electrónica: pasquale.cerbone@ute.edu.ec Orcid https://orcid.org/0000$\underline{0002-2871-4238}$
}

Artículo recibido: 16 de octubre, 2019

Enviado a corrección: 30 de enero, 2020

Aprobado: 20 de abril, 2020 


\section{Introducción}

Durante la última década el sistema de educación superior ecuatoriano ha experimentado una mejora en términos de calidad, debido sobre todo a una mayor inversión por parte del Gobierno en la educación y en la investigación (Vélez y Espinosa, 2018). Además, mediante la adopción de la Constitución del año 2008 y en cumplimiento del Art. 353 (Asamblea, 2011), y del Art. 15 de la Ley Orgánica de Educación Superior de 2010 (LOES), se conformó el Consejo de Educación Superior (CES) y el Consejo de Evaluación, Acreditación y Aseguramiento de la Calidad de la Educación Superior (CEAACES) (Asamblea, 2010), con capacidad de constituir entidades que se ocupen respectivamente de la "planificación, regulación y coordinación interna" (CES, 2018), y de los procesos relativos a la calidad de las instituciones pertenecientes al sistema de educación superior nacional (CACES, 2018). De esta forma, se sujetaron las universidades al cumplimiento de estándares de calidad en sus procesos internos de innovación, lo que ha causado una mejora del sistema en su totalidad.

Para tener una idea de la eficacia de estas reformas y, de acuerdo con el rango Webometrics $^{3,}$ (2018), podemos señalar que de 11.994 universidades analizadas a nivel global, la Universidad San Francisco de Quito se ubica en el puesto 1.757 y la Escuela Superior Politécnica del Litoral en el lugar 1.988; mientras que a nivel regional, entre 3742 universidades analizadas, las dos anteriores universidades ecuatorianas se ubican en el puesto 85 y 100, respectivamente. Por su parte, la Pontificia Universidad Católica del Ecuador ocupa el lugar 128, Universidad Técnica Particular de Loja se ubica en el puesto 142 y la Escuela Politécnica Nacional ocupa el lugar 149. Estos datos muestran que el posicionamiento de las universidades más importantes no es negativo. Vale agregar que la investigación realizada a nivel nacional ha mejorado de forma progresiva. Según el indicador Scimago Journal Rank (SJR, 2017), se ha pasado de 138 artículos citados en el año 2000, a un total de 2996 artículos citados en el año 2017, con la particularidad de que la producción científica mayormente reconocida corresponde a las ciencias exactas (informáticas, medicina, ingeniería y ciencias biológicas), lo que demuestra avances desde el punto de vista tecnológico. Lo descrito, aunque resulta alentador, no es suficiente para alcanzar los

3 (El Ranking Web de Centros de Investigación del Mundo es una iniciativa del Laboratorio de Cibermetría que pertenece al Instituto de Bienes y Políticas públicas (IPP), y que es parte del mayor centro nacional de investigación de España, el CSIC. Realiza análisis cuantitativo de Internet y de los contenidos de la red, especialmente de aquellos relacionados con el proceso de generación y comunicación académica del conocimiento científico). 
niveles de calidad que la educación superior debe ofrecer, si quiere convertirse en la impulsora de la transformación del país y del salto que este debe dar al mundo del desarrollo y del bienestar de la población. Allí, justamente, radica la importancia de esta investigación, con la cual se busca conocer el criterio del estudiantado, en el afán de entender su sentir y poder colegir las acciones que permitan guiar la inversión en temas de innovación. Aunado a ello, se busca orientar los esfuerzos para mejorar el contexto de la educación superior.

Lo expresado se torna más significativo debido a la situación económica nacional, que podría comprometer el avance del trabajo académico, pues la disminución de los ingresos estatales y la alta tasa de endeudamiento externo a corto y largo plazo, obligó al actual Gobierno a reducir el presupuesto del Estado y a declarar una eventual baja del 10\% en el presupuesto destinado a la educación superior pública durante el año 2019 (El Comercio, 2018). A ello hay que agregar que el Gobierno aprobó el Proyecto de ley Orgánica Reformatoria a la Ley Orgánica de Educación Superior (Presidencia de la República, 2018), en el cual se establece que los institutos tecnológicos superiores pueden ofertar, al igual que las otras instituciones de educación superior (IES), títulos de tercer y cuarto nivel, lo cual convierte a estos en competencia para las universidades privadas y cofinanciadas, debido a su oferta de servicios más baratos.

Esto demuestra la necesidad de una reforma del sistema educativo, en el sentido de permitir a los actores involucrados una competencia con estándares siempre más altos. Sin embargo, eso es posible solo a través de una racionalización del gasto, para que la innovación se dirija hacia objetivos correspondientes con las necesidades reales. De ahí que esta investigación tenga como objetivo general: conocer cuál es la percepción de quienes estudian en las universidades de la provincia de Pichincha, Ecuador, acerca de la innovación que sus instituciones desarrollan según los recursos puestos a disposición del estudiantado.

Asimismo, los objetivos específicos de la investigación fueron: establecer si existen diferencias de percepción entre estudiantes pertenecientes a diferentes tipos de universidades, verificar si existen diferencias de percepción entre estudiantes pertenecientes a diferentes tipos de facultades y, determinar, de forma general, cuáles son los recursos puestos a disposición por las universidades para los cuales mayormente se percibe necesiten de innovación.

El artículo se desarrolla a partir del fundamento teórico que respalda la investigación, el cual se construyó con fuentes bibliográficas específicas, que identifican las variables objeto de estudio. A esto se suma la descripción de la metodología empleada, con las unidades de 
análisis, las técnicas de recolección de datos y los resultados de su procesamiento. Todo esto conduce al apartado de resultados, con el análisis respectivo. En cuanto a las conclusiones, los resultados del estudio serán contrastados con la observación directa de la realidad por parte de los investigadores, en relación a la gestión del sistema universitario nacional por parte de las entidades públicas.

\section{Referente teórico}

Innovar es quizá uno de los más grandes desafíos que tienen las organizaciones en la actualidad. Este término entró en boga hace ya algunas décadas y tiene varias connotaciones, que coinciden en entenderlo como un elemento de diferenciación, que agrega valor a la organización (Gros y Lara, 2009). Para esta investigación es necesario contextualizar tal concepto en el ámbito universitario, el cual acoge diferentes áreas según la especialidad que abarca cada carrera. Por tanto, los criterios sobre innovación en educación superior van desde aquellos que aparecen como obvios, hasta los relacionados con la introducción de nuevas tecnologías (Charamba y Mutasa, 2018; González-Yebra; Aguilar; Aguilar y Matheu, 2018), y aquellos que consideran a la innovación, en la educación superior, como generadora de crecimiento económico de los países (Zhou y Luo, 2018).

En la literatura revisada hay coincidencias en cuanto a que la innovación en educación proporciona elementos que generan soluciones a problemas de la vida real (Thorsteinsson, 2013). No obstante, es necesario mirar más allá de la aparente quintaesencia de la tecnología y entender la innovación en la educación como un proceso en el que intervienen otras variables, tales como: el contexto, la cultura, las prácticas educativas (Ferrés y Masanet, 2018), todo lo cual nos lleva a abordar el tema desde una perspectiva amplia e integral. Así, Le (2018) considera que debe existir un proceso político que irrumpa en las nociones de autoridad y en las creencias implícitas sobre cómo enseñar y aprender, de tal forma que pueda reformularse la formación que recibe el estudiantado, a la vez que se propician cambios en el contexto social nacional.

Loaiza-Aguirre, Andrade-Abarca y Salazar-Romero (2017) sugieren que es necesario involucrar a las personas actoras de la educación superior en los procesos de innovación, dado que, de acuerdo a Cai (2017), la innovación incluye factores económicos, sociales, políticos, organizativos, institucionales y otros que influyen en su difusión y uso. Por tanto, resulta poco probable pretender aplicar modelos que han funcionado en otro contexto académico, sin considerar las experiencias y la cultura de la organización (Evenhouse et al., 
2018), más aún si consideramos que las nuevas prácticas generalmente requieren de cambios en los conocimientos, la identidad y las creencias de los maestros con respecto al aprendizaje y al alumnado (Niesz y Ryan, 2018).

Se debe tener presente que el entorno social y el clima organizacional de la institución educativa son parte del proceso de aprendizaje. Así, los aspectos administrativos, un ambiente favorable a la innovación y el apoyo de las autoridades pueden mejorar la actividad docente; es decir, el ambiente de aprendizaje y el proceso de aprendizaje se influyen mutuamente en una interacción continua (Solheim, Ertesvåg, y Dalhaug, 2018).

Por otra parte, es importante considerar las presiones administrativas a las que está sujeto el profesorado, ya que en muchos casos lo que logra implementar se ve afectado por limitaciones de tiempo, y esto genera tensiones o conflictos que reducen su creatividad. Es necesario entonces, otorgarle un mayor grado de libertad y reducir su carga administrativa, para mejorar el diseño de cursos y la calidad de la enseñanza (Lawrence, 2017). De ahí la necesidad de invertir en la capacitación y el apoyo continuo al equipo docente (Croda y López, 2016) para alcanzar mayor compromiso con la enseñanza, la pedagogía educativa y el apoyo al alumnado (Pinder y Shabbits, 2018). Se debe entender que los cambios tecnológicos requieren a la vez un cambio en el pensamiento del equipo docente y una apertura hacia nuevas formas de realizar su trabajo (Murillo-Ribes, Riaño-Galán y BerbelGómez, 2018), que le anime a asumir los riesgos que la experimentación conlleva y a desarrollar la voluntad para explorar (Sinclair y Aho, 2017), lo cual no siempre es fácil en vista de la alta resistencia al cambio y a la innovación (Leat y Thomas, 2018).

Invertir en infraestructura resulta también importante, pues, como señala González (2015), las instituciones de educación superior deben ofrecer infraestructura adecuada que permita aplicar, en las aulas, las políticas de innovación generadas. Aquí aparece otra directriz de los procesos de innovación, pues no se debe olvidar que tales procesos y políticas deben contener estrategias de sensibilización, para alcanzar una enseñanza inclusiva en los diversos cursos y ambientes universitarios, que se vuelva accesible y flexible a estudiantes mujeres y varones, con espacios en los cuales todos tengan acceso técnico y pedagógico a los recursos disponibles (de Anna y Covelli, 2018; de Ana y Della Volpe, 2011).

Con todos estos elementos se colige que, la implementación del uso de las TIC y de los entornos virtuales de aprendizaje, se convierte en herramienta prioritaria para la mejora de la calidad docente. Además, dicha implementación debe centrarse en tres ejes: la formación de la totalidad del profesorado en e-innovación; el fortalecimiento del trabajo 
colaborativo entre quienes imparten una misma asignatura o módulo y, el asegurar la estabilidad a los grupos de innovación (Martínez-Usarralde, López-Martín, Ramón; PérezCarbonell, 2018).

Esta primera aproximación a lo que significan los procesos de cambio y la introducción de actividades innovadoras en el quehacer educativo, nos conduce a reflexionar sobre los logros que, a través de ello, se pueden alcanzar, en especial si consideramos que actualmente los seres humanos vivimos interconectados a través de Internet, redes en línea y comunicación móvil (Barbosa y Amariles, 2019). Por ejemplo, países que son potencias desarrolladas se han centrado en reforzar la investigación y la educación, como una forma de mantener una ventaja competitiva con el resto del mundo, mientras de manera paralela estimulan la innovación para crear empleos y, por tanto, riqueza a nivel nacional (Ellison y Allen, 2016).

En este contexto, la investigación adopta una relevancia indiscutible (Rubio, 2012), pues como afirma Friberg (2018), si se quiere construir procesos de innovación es necesario invertir en educación, investigación, desarrollo técnico y tecnología de las comunicaciones, y así conectar a los sistemas nacionales de investigación entre sí, con incremento de la colaboración entre investigadores. Esta colaboración debe llevarnos a pensar en la organización de redes de investigación, que permita la convergencia exitosa de tecnologías para generar innovación y la superación del actuar individualista sin consensos (Hernández y Navarro, 2018). Dicho de otro modo, se debe trabajar para socializar la innovación (Kim y Jang, 2017; Montalvo, 2011). Esta idea refuerza el pensamiento de Hod, Bielaczyc y Ben-zvi (2018), quienes consideran que uno de los problemas más grandes que enfrenta la sociedad es superar divisiones y aprender a vivir y a trabajar de forma conjunta, en donde la diversidad sea una fortaleza. Es este concepto el que debe llevarse a la organización de las comunidades de aprendizaje, lo cual permite ofrecer una educación innovadora y construir conocimiento. Una concepción holística de la innovación educativa permitirá alcanzar los dos modos de innovación a los que hacen referencia Ellison y Allen (2016): por un lado, la innovación estructural, que comprende una transformación de la organización escolar y abarca los modelos y funciones administrativas; por otro lado, la innovación pedagógica que engloba la transformación del currículo, las prácticas y experimentos en el aula.

Finalmente, el estudio que se presenta a continuación pone en evidencia un aspecto importante de la innovación, es decir: la creación de valor para los beneficiarios de la misma (Teece, 2010). En este sentido, con el fin de analizar el nivel de innovación de las IES, como 
se ha dicho en la sección anterior, se partirá desde la percepción, de quienes estudian diferentes carreras, sobre el nivel de adecuación de las herramientas puestas a disposición por las universidades que frecuentan. Esto constituye el elemento de originalidad del estudio, en cuanto pone énfasis, por primera vez, en la visión interna acerca de las necesidades de innovación, en lugar de analizar el tema bajo el punto de vista de actores externos al sistema.

\section{Metodología y enfoque}

El estudio que se propone tiene un enfoque de tipo cuantitativo. Al ser de tipo descriptivo no conlleva la generación de hipótesis.

Las preguntas que guían la investigación son las siguientes:

1) ¿Cuál es la percepción que los estudiantes universitarios residentes en la provincia de Pichincha (Ecuador) tienen sobre el nivel de adecuación de los recursos puestos a disposición por su universidad?

2) ¿Cuáles son los elementos que explican dicha percepción?

3) ¿Existe una diferencia de percepción entre estudiantes pertenecientes a diferentes tipos de facultades?

4) ¿Existe una diferencia de percepción entre estudiantes pertenecientes a diferentes tipos de universidades?

La respuesta a la primera pregunta tendrá el fin de determinar qué ítems entre los analizados necesitan mayormente de innovación. La respuesta a la segunda tiene el fin de dar indicaciones a los organismos públicos sobre la correcta asignación de los recursos entre los diferentes tipos de facultades. La tercera respuesta, en cambio, se propone generar una reflexión sobre el eventual desnivel que puede existir entre los diferentes tipos de universidades, si existe una diferente percepción entre sus estudiantes.

Los resultados que se presentan en este artículo constituyen solo una parte de los que se extrapolaron del estudio denominado "Percepción sobre las necesidades de innovación al interno de las instituciones de educación superior de la provincia de Pichincha, Ecuador", que se realizó durante marzo y mayo de 2019.

El estudio se basó en un instrumento de encuesta, cuyas categorías y variables se definieron sobre la base de literatura existente en temas de innovación en las IES, que se presentó en el marco teórico de este artículo y que fueron validadas por un panel de docentes conocedores del tema de este estudio. En cuanto al posicionamiento de las 
preguntas a lo interno de la encuesta y al tipo de respuesta a utilizarse, se acogió el criterio de un docente experto en estadística. Además, mediante un análisis factorial (anexo 1) se demuestra la existencia de una correlación entre las variables identificadas para cada categoría, lo que significa que el instrumento utilizado puede considerarse válido.

La encuesta evaluó la percepción sobre la necesidad de innovación de cuatro categorías de variables: i) infraestructura, tecnología y recursos, ii) estructura organizacional, iii) personas y competencias profesionales, iv) plus ofertados por la institución (con lo cual se entiende el conjunto de elementos que diferencian las universidades, en el ámbito de la oferta formativa). El tipo de respuesta que se eligió para cada pregunta fue la escala Likert con valores de 1 a 5, correspondientes a las respuestas "totalmente inadecuado", “inadecuado", "ni inadecuado, ni adecuado", "adecuado" y "totalmente adecuado".

\subsection{Unidades de análisis}

Para el cálculo de la muestra se consideró como universo el total de estudiantes universitarios de la provincia de Pichincha (Ecuador), en donde se concentran 20 universidades. Además, considerando la diferencia de necesidades entre estudiantes de facultades de ciencias sociales, educativas, de artes y humanidades, periodismo e información, negocios, administración y jurisprudencia (que desde ahora en adelante llamaremos "Tipo 1" para facilitar la descripción de los resultados), y de ciencias exactas y naturales, de tecnología de información, ingeniería, agricultura, veterinarias y médicas ${ }^{4}$ (que desde ahora en adelante llamaremos “Tipo 2") (Unesco, 2015), se decidió adoptar dos muestreos probabilísticos para cada categoría de estudiantes.

\subsection{Técnicas de recolección de datos}

El levantamiento de información tuvo lugar durante los meses de enero y marzo de 2019, en la provincia de Pichincha (Ecuador), al interior de las universidades presentes en el territorio. La encuesta fue aplicada de manera personal, de acuerdo a una estratificación por sexo, tipo de facultad universitaria (según la división considerada en el párrafo anterior) y tipo de universidad (pública, privada o cofinanciada), considerando estos factores como importantes para un análisis profundo del tema.

\footnotetext{
4 Para la determinación de las carreras a insertarse en la una o en la otra categoría se hizo referencia al International Standard Classification of Education de la Unesco.
} 
Después de la recolección de la información y su tabulación, se depuró el conjunto de datos, de los eventuales errores de redacción o de la información que los entrevistados no quisieron otorgar. Luego, se realizó un análisis descriptivo, que contiene el cálculo de la media y la relativa desviación típica, con el fin de tener una visión clara, sobre la percepción de las variables objeto de estudio $\mathrm{y}$, finalmente, teniendo como variable explicada la pregunta: "¿Generalmente cómo percibe los recursos puestos a disposición por su universidad?", se generó un modelo de regresión lineal múltiple, a través del cual se estudió la percepción de 6 categorías de entrevistados: 1) Estudiantes de Tipo 1 inscritos en universidades públicas; 2) Estudiantes de Tipo 2 inscritos en universidades públicas; 3) Estudiantes de Tipo 1 inscritos en universidades cofinanciadas; 4) Estudiantes de Tipo 2 inscritos en universidades cofinanciadas; 5) Estudiantes de Tipo 1 inscritos en universidades privadas; 6) Estudiantes de Tipo 2 inscritos en universidades privadas. Todo ello con el fin de establecer si existen variables que expliquen detalladamente, su percepción.

La selección de las variables del modelo ocurrió de acuerdo a la técnica de la eliminación progresiva manual, según al nivel de significancia (Rojo, 2007).

Se eligió la técnica del modelo de regresión lineal múltiple, pues la propuesta del artículo no consiste solo en la identificación de las diferencias en las opiniones de los estudiantes pertenecientes a las dos ramas del conocimiento (para esto un modelo del tipo Anova habría sido más adecuado), sino que se quiso identificar también el conjunto de elementos que puede explicar la opinión observada.

El fin del estudio es doble: tener una visión general del nivel de innovación percibido por los estudiantes y entender si existe una diferencia significativa entre quienes estudian en distintos tipos de universidades, para generar una reflexión global sobre el modelo educativo superior ecuatoriano. Para la evaluación del modelo de regresión se verificaron previamente todos los supuestos necesarios, y se utilizó la escala de validación propuesta por Rojo $(2007)^{5}$, que se basa en la observación del coeficiente de determinación ajustado.

\subsection{Procesamiento de análisis}

Para una más ágil comprensión de los resultados, inicialmente se codificaron las variables como se muestra a continuación en la tabla 1.

${ }^{5}(<0.3=$ muy malo, $0.3-0.4=$ malo, $0.4-0.5=$ regular, $0.5-0.85=$ bueno, $>0.85=$ sospechoso $)$. 
Tabla 1

Código, variable y categoría

\begin{tabular}{|c|c|c|}
\hline Código & Variable & Categoría \\
\hline AU & Aulas & Infraestructura, tecnología y recursos \\
\hline LAB & Laboratorios & Infraestructura, tecnología y recursos \\
\hline $\mathrm{BI}$ & Biblioteca & Infraestructura, tecnología y recursos \\
\hline OEC & Otros espacios & Infraestructura, tecnología y recursos \\
\hline EE & Equipos electrónicos & Infraestructura, tecnología y recursos \\
\hline PI & Programas informáticos & Infraestructura, tecnología y recursos \\
\hline $\mathrm{Cl}$ & Conexión a internet & Infraestructura, tecnología y recursos \\
\hline AP & Acceso a plataformas & Infraestructura, tecnología y recursos \\
\hline PC & Planificación de los cursos & Estructura organizacional \\
\hline PT & Planificación del tiempo & Estructura organizacional \\
\hline OU & Organización de la universidad & Estructura organizacional \\
\hline $\mathrm{MC}$ & Manejo de los cambios & Estructura organizacional \\
\hline GC & Gestión de la comunicación & Estructura organizacional \\
\hline TD & Toma de decisiones & Estructura organizacional \\
\hline OP & Optimización de los procesos & Estructura organizacional \\
\hline $\mathrm{CO}$ & Cultura organizacional & Estructura organizacional \\
\hline IN & Investigación & Estructura organizacional \\
\hline GU & Gestión de la universidad & Estructura organizacional \\
\hline RD & Relación con los docentes & Personas, competencias profesionales \\
\hline PD & Preparación de los docentes & Personas, competencias profesionales \\
\hline MD & Material usado por los docentes & Personas, competencias profesionales \\
\hline MDO & Metodología de docencia & Personas, competencias profesionales \\
\hline CAS & Contenidos de las asignaturas & Personas, competencias profesionales \\
\hline ABM & Acceso a becas por mérito & Plus ofertados por la institución \\
\hline AEX & Actividades extracurriculares & Plus ofertados por la institución \\
\hline OFD & $\begin{array}{l}\text { Otras formas de difusión del } \\
\text { conocimiento }\end{array}$ & Plus ofertados por la institución \\
\hline CON & Convenios manejados por la universidad & Plus ofertados por la institución \\
\hline OPR & Opiniones propuestas & Plus ofertados por la institución \\
\hline $\mathrm{CCl}$ & Colaboración con otras instituciones & Plus ofertados por la institución \\
\hline GR & $\begin{array}{l}\text { Percepción general sobre recursos de la } \\
\text { universidad }\end{array}$ & Variable explicada \\
\hline
\end{tabular}

Elaborado por los autores a partir del estudio "Percepción sobre las necesidades de innovación al interno de las instituciones de educación superior de la provincia de Pichincha, Ecuador".

A continuación, en la tabla 2 se muestra los datos relativos al análisis descriptivo 
Tabla 2

Análisis descriptivo de la percepción de quienes estudian en universidades públicas

\begin{tabular}{|c|c|c|c|c|c|c|c|c|c|c|c|c|c|c|c|c|c|c|}
\hline \multicolumn{18}{|c|}{ Universidades cofinanciadas } & \\
\hline & Tipo & & Tipo & & & Tipo & & & Tipo & & & Tipo & & & Tipo & & & \\
\hline $\mathbf{N}$ & $\mu$ & $\sigma$ & $\mathbf{N}$ & $\boldsymbol{\mu}$ & $\sigma$ & $\mathbf{N}$ & $\boldsymbol{\mu}$ & $\sigma$ & $\mathbf{N}$ & $\boldsymbol{\mu}$ & $\sigma$ & $\mathbf{N}$ & $\boldsymbol{\mu}$ & $\sigma$ & $\mathbf{N}$ & $\mu$ & $\sigma$ & \\
\hline$A U$ & 126 & 3,36 & 1,15 & 132 & 3,08 & 1,06 & 136 & 3,97 & ,83 & 126 & 4,03 & ,91 & 128 & 3,87 & ,86 & 119 & 3,97 & ,81 \\
\hline LAB & 126 & 3,41 & 1,22 & 132 & 3,46 & 1,46 & 136 & 4,11 & ,96 & 126 & 4,03 & ,91 & 128 & 4,07 & ,81 & 119 & 4,07 & ,91 \\
\hline BI & 126 & 3,56 & 1,11 & 132 & 3,40 & 1,29 & 136 & 3,89 & ,88 & 126 & 3,80 & ,96 & 128 & 3,88 & ,80 & 119 & 3,84 &, 91 \\
\hline OEC & 126 & 2,97 & 1,15 & 132 & 3,18 & 1,12 & 136 & 3,54 & 1,06 & 126 & 3,74 & 1,07 & 128 & 3,52 & 1,11 & 119 & 3,50 & 1,09 \\
\hline EE & 126 & 3,23 & 1,14 & 132 & 3,20 & 1,32 & 136 & 3,69 &, 97 & 126 & 3,65 & 1,02 & 128 & 3,87 & ,85 & 119 & 3,93 & ,83 \\
\hline PI & 126 & 3,14 & 1,18 & 132 & 3,15 & 1,21 & 136 & 3,62 & ,99 & 126 & 3,59 & 1,07 & 128 & 3,83 & ,86 & 119 & 3,94 & 80 \\
\hline $\mathrm{Cl}$ & 126 & 3,20 & 1,11 & 132 & 3,08 & 1,22 & 136 & 3,36 & 1,34 & 126 & 3,40 & 1,20 & 128 & 3,75 & 1,13 & 119 & 3,98 & ,90 \\
\hline AP & 126 & 3,42 & 1,05 & 132 & 3,16 & 1,27 & 136 & 3,88 & ,88 & 126 & 3,73 & 1,05 & 128 & 3,82 & 1,05 & 119 & 4,00 & ,88 \\
\hline RD & 126 & 3,26 & 1,10 & 132 & 3,15 & 1,10 & 136 & 4,04 & ,90 & 126 & 4,02 & ,99 & 128 & 3,78 & ,82 & 119 & 3,81 & ,88 \\
\hline PC & 126 & 3,29 & 1,03 & 132 & 3,06 & 1,04 & 136 & 3,70 & ,96 & 126 & 3,79 & 1,00 & 128 & 3,67 & ,98 & 119 & 3,51 & ,82 \\
\hline PT & 126 & 3,37 & 1,00 & 132 & 3,49 & ,96 & 136 & 3,93 & ,87 & 126 & 3,99 & ,88 & 128 & 3,86 & ,85 & 119 & 3,89 & ,74 \\
\hline OU & 126 & 2,95 & 1,12 & 132 & 3,17 & 1,15 & 136 & 3,58 & 1,09 & 126 & 3,75 & 1,07 & 128 & 3,55 & 1,03 & 119 & 3,63 & ,96 \\
\hline MC & 126 & 3,17 & 1,20 & 132 & 3,27 & 1,24 & 136 & 3,52 & 1,09 & 126 & 3,69 & 1,12 & 128 & 3,55 & 1,12 & 119 & 3,76 & 1,07 \\
\hline GC & 126 & 3,07 & 1,02 & 132 & 3,12 & 1,23 & 136 & 3,57 & 1,04 & 126 & 3,63 & 1,03 & 128 & 3,46 & 1,10 & 119 & 3,73 & 1,10 \\
\hline TD & 126 & 2,92 & 1,08 & 132 & 3,20 & 1,25 & 136 & 3,44 & 1,29 & 126 & 3,51 & 1,09 & 128 & 3,61 & 1,12 & 119 & 3,52 & 1,17 \\
\hline OP & 126 & 3,03 & 1,15 & 132 & 3,15 & 1,25 & 136 & 3,61 & 1,12 & 126 & 3,62 & 1,00 & 128 & 3,77 & 1,09 & 119 & 3,89 & 1,19 \\
\hline $\mathrm{CO}$ & 126 & 3,16 & 1,10 & 131 & 3,30 & 1,10 & 136 & 3,44 & 1,05 & 126 & 3,77 & ,98 & 128 & 3,60 & 1,25 & 119 & 3,58 & 1,10 \\
\hline IN & 126 & 3,43 & 1,24 & 132 & 3,28 & 1,04 & 136 & 3,81 & 1,09 & 126 & 3,73 & 87 & 128 & 3,94 &, 98 & 119 & 3,78 &, 919 \\
\hline GU & 126 & 3,15 &, 98 & 132 & 3,22 & 1,03 & 136 & 3,66 & 1,00 & 126 & 3,81 & ,93 & 128 & 3,76 & 1,04 & 119 & 3,61 & 1,11 \\
\hline PD & 126 & 3,88 & 1,00 & 132 & 3,78 & 1,07 & 136 & 4,06 & ,83 & 126 & 4,02 & ,95 & 128 & 4,26 &, 78 & 119 & 4,16 & 84 \\
\hline MD & 126 & 3,38 & 1,10 & 132 & 3,50 & 1,08 & 136 & 3,82 & ,98 & 126 & 4,02 & ,92 & 128 & 3,92 &, 91 & 119 & 4,00 & ,92 \\
\hline MDO & 126 & 3,37 & ,93 & 132 & 3,83 & 4,61 & 136 & 3,75 & ,89 & 126 & 3,92 & ,94 & 128 & 4,06 & ,80 & 118 & 3,94 & ,81 \\
\hline CAS & 126 & 3,56 & 1,04 & 132 & 3,55 & 1,06 & 136 & 3,83 & ,93 & 126 & 3,96 & ,91 & 128 & 3,93 & ,88 & 119 & 3,81 & ,88 \\
\hline ABM & 126 & 3,57 & 1,44 & 132 & 3,55 & 1,30 & 136 & 3,86 & 1,09 & 126 & 4,11 & ,91 & 128 & 3,85 & 1,01 & 119 & 3,78 & 1,11 \\
\hline AEX & 126 & 3,12 & 1,35 & 132 & 3,21 & 1,27 & 136 & 3,66 & 1,08 & 126 & 3,65 & 1,16 & 128 & 3,60 & 1,11 & 119 & 3,88 & 1,06 \\
\hline OFD & 126 & 3,32 & 1,29 & 132 & 3,30 & 1,20 & 136 & 3,66 & 1,17 & 126 & 3,81 & 1,18 & 128 & 3,89 & ,89 & 119 & 4,03 & ,95 \\
\hline CON & 126 & 3,48 & 1,25 & 132 & 3,37 & 1,19 & 136 & 3,67 & 1,28 & 126 & 3,77 & 1,07 & 128 & 4,00 & ,99 & 119 & 4,02 & ,97 \\
\hline OPR & 126 & 3,31 & 1,19 & 132 & 3,23 & 1,04 & 135 & 3,56 & 1,10 & 126 & 3,89 & 1,07 & 128 & 3,54 & 1,07 & 119 & 3,85 & 1,00 \\
\hline $\mathrm{CCl}$ & 126 & 3,38 & 1,36 & 132 & 3,25 & 1,17 & 136 & 3,35 & 1,17 & 126 & 3,80 & 1,17 & 128 & 3,53 & 1,09 & 119 & 3,67 & 1,06 \\
\hline GR & 126 & 3,50 & 1,13 & 132 & 3,29 &, 95 & 136 & 3,71 & 1,01 & 126 & 3,93 & ,96 & 128 & 3,83 & ,88 & 119 & 4,00 &, 95 \\
\hline
\end{tabular}

Elaborado por los autores a partir del estudio "Percepción sobre las necesidades de innovación al interno de las instituciones de educación superior de la provincia de Pichincha, Ecuador". 
La tabla muestra una clara diferencia de percepción entre el estudiantado de los diferentes tipos de universidades. Particularmente: el estudiantado de Tipo 1 y 2 de universidades públicas muestra un promedio inferior a 4 para todas las variables consideradas al interno de la encuesta. En el caso del estudiantado de universidades cofinanciadas y privadas, en cambio, algunas variables muestran una evaluación promedia superior a 4. Cabe destacar que el estudiantado de las universidades privadas muestra una calificación promedio, superior a 4 , en un mayor número de casos frente al estudiantado de las otras dos categorías universitarias.

A continuación, se muestra los resultados de los modelos de regresión lineal múltiple (tabla 3 , tabla 4 , tabla 5 , tabla 6 , tabla 7 y tabla 8 ).

\section{Tabla 3}

Modelo de regresión lineal múltiple de la percepción del estudiantado Tipo 1 en universidades públicas

\begin{tabular}{lcccc}
\hline Modelo & $\mathrm{R}$ & $\begin{array}{c}\mathrm{R} \\
\text { cuadrado }\end{array}$ & $\begin{array}{c}\text { R cuadrado } \\
\text { ajustado }\end{array}$ & Error estándar de la estimación \\
\hline 1 &, $734^{\mathrm{a}}$ &, 539 &, 527 &, 78136
\end{tabular}

a. Predictores: (Constante), CCI, MD, OFD

\begin{tabular}{|c|c|c|c|c|c|c|}
\hline \multirow{2}{*}{\multicolumn{2}{|c|}{ Modelo }} & \multicolumn{2}{|c|}{ Coeficientes no estandarizados } & \multicolumn{3}{|l|}{$\begin{array}{c}\text { Coeficientes } \\
\text { estandarizados }\end{array}$} \\
\hline & & B & Desv. Error & Beta & $\mathrm{t}$ & Sig. \\
\hline 1 & Constante &, 513 & ,262 & & 1,955 & ,053 \\
\hline & MD & ,394 & ,068 & ,384 & 5,813 & ,000 \\
\hline & OFD & 219 & ,063 & ,250 & 3,483 & ,001 \\
\hline & $\mathrm{CCl}$ & 273 & ,060 & 328 & 4,564 & ,000 \\
\hline
\end{tabular}

a. Variable dependiente: GR

Elaborado por los autores a partir del estudio "Percepción sobre las necesidades de innovación al interno de las instituciones de educación superior de la provincia de Pichincha, Ecuador".

Tabla 4

Modelo de regresión lineal múltiple de la percepción del estudiantado Tipo 2 en públicas

\begin{tabular}{ccccc}
\hline Modelo & $\mathrm{R}$ & $\mathrm{R}$ cuadrado & $\begin{array}{c}\text { R cuadrado } \\
\text { ajustado }\end{array}$ & $\begin{array}{c}\text { Error estándar de la } \\
\text { estimación }\end{array}$ \\
\hline 1 &, $623^{\mathrm{a}}$ &, 388 &, 379 &, 75269
\end{tabular}

a. Predictores: (Constante), CCl, OFD, CON

\begin{tabular}{|c|c|c|c|c|c|c|}
\hline \multirow{2}{*}{\multicolumn{2}{|c|}{ Modelo }} & \multicolumn{2}{|c|}{ Coeficientes no estandarizados } & \multirow{2}{*}{$\begin{array}{c}\text { Coeficientes } \\
\text { estandarizados } \\
\text { Beta }\end{array}$} & \multirow[b]{2}{*}{$\mathrm{t}$} & \multirow[b]{2}{*}{ Sig. } \\
\hline & & B & Desv. Error & & & \\
\hline 1 & Constante & 1,384 & ,221 & & 6,258 &, 000 \\
\hline & OFD & 275 & 062 & ,346 & 4,431 &, 000 \\
\hline & $\mathrm{CCl}$ & ,308 &, 063 & ,380 & 4,859 &, 000 \\
\hline
\end{tabular}

a. Variable dependiente: GR

Elaborado por los autores a partir del estudio "Percepción sobre las necesidades de innovación al interno de las instituciones de educación superior de la provincia de Pichincha, Ecuador". 
En cuanto al estudiantado de universidades públicas de Tipo 1, se muestra que, la percepción general sobre los recursos puestos a disposición de la universidad, depende de las variables MD, OFD y CCI. El estudiantado de Tipo 2, en cambio, expresa una percepción que se explica sobre todo con las variables OFD y CCl.

\section{Tabla 5}

Modelo de regresión lineal múltiple de la percepción del estudiantado Tipo 1 en universidades cofinanciadas

\begin{tabular}{|c|c|c|c|c|}
\hline Modelo & $\mathrm{R}$ & $\begin{array}{c}\mathrm{R} \\
\text { cuadrado }\end{array}$ & $\begin{array}{l}\mathrm{R} \text { cuadrado } \\
\text { aiustado }\end{array}$ & $\begin{array}{c}\text { Error estándar de la } \\
\text { estimación }\end{array}$ \\
\hline 1 & $741^{a}$ & ,549 & ,542 & 68235, \\
\hline
\end{tabular}

a. Predictores: (Constante), OPR, CO, OFD, MD, PC

\begin{tabular}{|c|c|c|c|c|c|c|}
\hline \multirow[b]{2}{*}{ Modelo } & & \multicolumn{2}{|c|}{$\begin{array}{l}\text { Coeficientes no } \\
\text { estandarizados }\end{array}$} & Coeficientes estandarizados & \multirow[b]{2}{*}{$t$} & \multirow[b]{2}{*}{ Sig. } \\
\hline & & B & Desv. Error & Beta & & \\
\hline 1 & Constante & ,727 & 251 & & 2,898 & ,004 \\
\hline & PC & ,350 & ,080 & ,337 & 4,367 & ,000 \\
\hline & OPR & ,352 & ,070 & ,385 & 5,033 & ,000 \\
\hline
\end{tabular}

a. Variable dependiente: GR

Elaborado por los autores a partir del estudio "Percepción sobre las necesidades de innovación al interno de las instituciones de educación superior de la provincia de Pichincha, Ecuador".

\section{Tabla 6}

Modelo de regresión lineal múltiple de la percepción del estudiantado Tipo 2 en universidades cofinanciadas

\begin{tabular}{ccccc}
\hline Modelo & $\mathrm{R}$ & $\mathrm{R}$ cuadrado & $\begin{array}{c}\text { R cuadrado } \\
\text { ajustado }\end{array}$ & $\begin{array}{c}\text { Error estándar de la } \\
\text { estimación }\end{array}$ \\
\hline 1 &, $702^{\mathrm{a}}$ &, 493 &, 480 &, 69891
\end{tabular}

a. Predictores: (Constante), CON, RD, CI, MDO

\begin{tabular}{|c|c|c|c|c|c|c|}
\hline \multirow[b]{2}{*}{ Modelo } & & \multicolumn{2}{|c|}{ Coeficientes no estandarizados } & $\begin{array}{c}\text { Coeficientes } \\
\text { estandarizados }\end{array}$ & \multirow[b]{2}{*}{$\mathrm{t}$} & \multirow[b]{2}{*}{ Sig. } \\
\hline & & B & Desv. Error & Beta & & \\
\hline 1 & Constante & ,564 & ,316 & & 1,784 & 077 \\
\hline & $\mathrm{RD}$ & 278 & 077 & ,286 & 3,614 &, 000 \\
\hline & MDO & ,335 & 086 & ,326 & 3,906 &, 000 \\
\hline & CON & 249 & ,064 & ,275 & 3,873 & ,000 \\
\hline
\end{tabular}

a. Variable dependiente: GR

Elaborado por los autores a partir del estudio "Percepción sobre las necesidades de innovación al interno de las instituciones de educación superior de la provincia de Pichincha, Ecuador".

En cuanto al estudiantado de universidades cofinanciadas de Tipo 1, se muestra que, la percepción general sobre los recursos puestos a disposición de la universidad depende de las variables PC y OPR. El estudiantado de Tipo 2, en cambio, expresa una percepción que se explica sobre todo con las variables RD, MDO y CON. 
Tabla 7

Modelo de regresión lineal múltiple de la percepción del estudiantado Tipo 1 en universidades privadas

\begin{tabular}{ccccc}
\hline Modelo & $\mathrm{R}$ & $\mathrm{R}$ cuadrado & $\begin{array}{c}\text { R cuadrado } \\
\text { ajustado }\end{array}$ & $\begin{array}{c}\text { Error estándar de la } \\
\text { estimación }\end{array}$ \\
\hline 1 &, $726^{\mathrm{a}}$ &, 528 &, 508 &, 62093
\end{tabular}

a. Predictores: (Constante), CCI, IN, ABM, PT, OU

\begin{tabular}{|c|c|c|c|c|c|c|}
\hline \multirow[b]{2}{*}{ Model } & & \multicolumn{2}{|c|}{$\begin{array}{l}\text { Coeficientes no } \\
\text { estandarizados }\end{array}$} & \multirow{2}{*}{$\begin{array}{c}\text { Coeficientes } \\
\text { estandarizados } \\
\text { Beta }\end{array}$} & \multirow[b]{2}{*}{$\mathrm{t}$} & \multirow[b]{2}{*}{ Sig. } \\
\hline & & B & Desv. Error & & & \\
\hline 1 & Constante & ,143 & ,354 & & 405 & ,686 \\
\hline & PT & ,426 & ,074 & ,411 & 5,773 & ,000 \\
\hline & OU & -226 &, 064 &,- 264 & $-3,536$ & 001 \\
\hline & IN & 200 & ,059 & ,222 & 3,407 & ,001 \\
\hline & ABM & 203 & ,057 & ,233 & 3,539 &, 001 \\
\hline & $\mathrm{CCl}$ & ,360 & ,055 & ,445 & 6,577 & ,000 \\
\hline
\end{tabular}

a. Variable dependiente: GR

Elaborado por los autores a partir del estudio "Percepción sobre las necesidades de innovación al interno de las instituciones de educación superior de la provincia de Pichincha, Ecuador".

\section{Tabla 8}

Modelo de regresión lineal múltiple de la percepción del estudiantado Tipo 2 en universidades privadas

\begin{tabular}{ccccc}
\hline Modelo & $\mathrm{R}$ & $\begin{array}{c}\mathrm{R} \\
\text { cuadrado }\end{array}$ & $\begin{array}{c}\text { R cuadrado } \\
\text { ajustado }\end{array}$ & $\begin{array}{c}\text { Error estándar de la } \\
\text { estimación }\end{array}$ \\
\hline 1 &, $642^{\mathrm{a}}$ &, 412 &, 401 &, 73985
\end{tabular}

a. Predictores: (Constante), CCI, PD, OPR, MDO

\begin{tabular}{|c|c|c|c|c|c|c|}
\hline \multirow[b]{2}{*}{ Modelo } & & \multicolumn{2}{|c|}{ Coeficientes no estandarizados } & \multicolumn{3}{|l|}{ Coeficientes estandarizados } \\
\hline & & B & Desv. Error & Beta & $t$ & Sig. \\
\hline 1 & $\begin{array}{c}\text { (Constante } \\
\text { ) }\end{array}$ & ,700 & ,377 & & 1,854 & ,066 \\
\hline & MDO & ,495 & ,088 & ,422 & 5,598 & ,000 \\
\hline & OPR & ,352 & ,072 & 368 & 4,887 & ,000 \\
\hline
\end{tabular}

a. Variable dependiente: GR

Elaborado por los autores a partir del estudio "Percepción sobre las necesidades de innovación al interno de las instituciones de educación superior de la provincia de Pichincha, Ecuador".

En cuanto al estudiantado de universidades privadas de Tipo 1, se muestra que, la percepción general sobre los recursos puestos a disposición de la universidad depende de las variables $\mathrm{PT}, \mathrm{OU}, \mathrm{IN}, \mathrm{ABM}$ y $\mathrm{CCl}$. El estudiantado de Tipo 2, en cambio, expresa una percepción que se explica sobre todo con las variables MDO y OPR. 


\section{Resultados}

A partir del análisis descriptivo, un dato puede evidenciarse inmediatamente: en las universidades públicas la percepción general sobre los recursos puestos a disposición por las universidades es inferior al de otras categorías, con valores promedio de 3,5 para quienes estudian ciencias exactas y 3,29 para quienes estudian ciencias sociales. Las universidades cofinanciadas y privadas, en cambio (considerando el tipo de escala usada, que es muy breve), presentan valores netamente superiores, con un promedio de 3,71 y 3,83 para quienes estudian ciencias exactas y de 3,93 y 4,00 para quienes siguen ciencias sociales. Resalta también una particularidad: en el caso de las universidades públicas, la apreciación promedio sobre la variable "percepción general sobre recursos de la Universidad", por parte del alumnado de ciencias exactas es mayor frente al de ciencias sociales, mientras que en las otras universidades existe una realidad opuesta. Además, en el caso de la apreciación de estudiantes de ciencias exactas de universidades públicas, destacan de manera negativa, frente al promedio general, las variables: otros espacios, organización de la universidad y toma de decisiones, que presentan una calificación del tipo "inadecuado".

En el caso de las universidades cofinanciadas, en la apreciación de quienes estudian ciencias exactas, destacan positivamente las variables: laboratorios, relación de ambos sexos y su preparación, pues presentan un promedio mayor al resto, con una calificación del tipo "adecuado". Lo mismo ocurre en el caso de quienes estudian ciencias sociales y pertenecen a igual tipo de universidad, con las variables: aulas, laboratorios, relación con docentes tanto hombres como mujeres y su preparación, material usado por maestros y maestras, y acceso a becas por mérito.

En fin, en cuanto a las universidades privadas, la apreciación del alumnado de ciencias exactas es del tipo "adecuado", en lo relacionado a las variables: "laboratorios", "preparación de docentes tanto hombres como mujeres", "metodología de docencia" y "convenios manejados por la universidad", que destacan sobre el promedio general. Lo mismo ocurre con la apreciación de quienes estudian ciencias sociales en cuanto a las variables: "laboratorios", "acceso a plataformas", "preparación de docentes de ambos sexos", el "material" que usan, "otras formas de difusión del conocimiento" y "convenios manejados por la universidad".

En cuanto a los modelos de regresión, hay que aclarar que, con base en la escala de validación propuesta en la metodología, el modelo que se refiere a la percepción del alumnado de ciencias sociales de las universidades públicas es "malo", lo que indica que la apreciación general de esta categoría no puede ser explicada exhaustivamente por ninguna 
de las variables analizadas. En los demás casos los modelos son válidos y se los puede considerar "buenos". Particularmente, en el caso de quienes estudian ciencias exactas de universidades públicas, la apreciación general sobre los recursos puestos a disposición por parte de su universidad, se explica con las variables "planificación de cursos" y "opiniones propuestas", que presentan un peso relativo (B) muy bajo. Esto significa que la apreciación general sobre la variable "percepción general sobre recursos de la universidad", que es del tipo "ni inadecuado, ni adecuado", encuentra una tendencia similar constante entre los entrevistados en las variables antes mencionadas. La misma lectura puede darse al modelo referente a la apreciación de: i) estudiantes de ciencias exactas de universidades cofinanciadas, explicado por las variables: "relación con docentes de ambos sexos", "metodología de docencia", y "convenios manejados por la universidad"; ii) estudiantes de ciencias sociales de universidades cofinanciadas, que es explicado por las variables: "planificación del tiempo", "organización de la universidad", "investigación”, "acceso a becas por mérito" y "colaboración con otras instituciones"; iii) estudiantes de ciencias exactas de universidades privadas, que presentan como variables predictoras: "planificación del tiempo", "organización de la universidad”, "investigación”, “acceso a becas por mérito” y "colaboración con otras instituciones"; iv) estudiantes de ciencias sociales de universidades privadas que presentan como variables predictoras: "metodología de docencia” y "opiniones propuestas".

\section{Conclusiones}

La respuesta a la primera pregunta planteada por este estudio, y que se refiere a su objetivo general, evidencia que la apreciación general de los estudiantes sobre el nivel de adecuación de los recursos puestos a disposición por sus universidades, es del tipo "ni adecuado, ni inadecuado".

Con relación al primer objetivo específico se puede evidenciar que existe una diferente percepción entre estudiantes de facultades distintas, aunque mínima. Particularmente, en el estudiantado de Tipo 1, en las universidades públicas se manifiesta un grado de insatisfacción más alto que en todos los demás ámbitos analizados, en cuanto al grado de adecuación de los recursos puestos a disposición por la universidad; aquí se genera la idea de que la universidad pública tiene que innovar, sobre todo en cuanto a los recursos, a disposición de esta particular categoría de estudiantes. Es diferente en el caso de estudiantes de ciencias sociales de universidades privadas y cofinanciadas, cuya percepción, al ser analizada, 
manifiesta una diferencia con estudiantes de ciencias exactas, quienes demuestran más exigencia en cuanto a los recursos disponibles.

En respuesta al segundo objetivo específico, se evidencia que la percepción de quienes estudian en universidades públicas es, en general, diferente a la de quienes lo hacen en las universidades cofinanciadas y privadas, en relación con las actividades inherentes a la innovación desarrolladas en sus centros de estudio. A partir de la observación directa de la realidad por parte de los autores, se puede explicar esta situación mediante las dificultades que tiene la universidad pública, acerca de procesos burocráticos y presupuesto, ya que depende de los recursos asignados por el Estado, lo que podría significar una limitante con la que no bregan las instituciones privadas.

Finalmente, con respecto al último objetivo específico, se puede observar que las variables que mayormente explican el grado de satisfacción expresado por los estudiantes, se relacionan con la categoría "organización de la universidad", lo que deja concluir que es recomendable dirigir la inversión hacia la innovación de los demás recursos incluidos en las categorías analizadas.

La infraestructura de innovación es lo que más observan y valoran quienes estudian en el nivel superior de enseñanza, pues con ello tienen contacto prácticamente desde el primer acercamiento a la universidad y a lo largo de su carrera. Algo similar sucede con los profesores y las profesoras, de quienes, al tener trato permanente, valoran el tipo de relación que desarrollan, su preparación en temas de innovación, así como la metodología y el material innovador que emplean.

\section{Referencias}

Asamblea Nacional. (2010). Ley Orgánica de Educación Superior. Recuperado de https://procuraduria.utpl.edu.ec/sitios/documentos/NormativasPublicas/LEY\%20ORG\%C 3\%81NICA\%20REFORMATORIA\%20A\%20LEY\%20ORG\%C3\%81NICA\%20DE\%20ED UCACI\%C3\%93N\%20SUPERIOR\%2002-08-2018.pdf

Asamblea Nacional Constituyente. (2011). Constitución de la República del Ecuador. Recuperado de https://www.oas.org/juridico/pdfs/mesicic4_ecu_const.pdf

Barbosa, Sergio., y Amariles, Marlly. (2019). Learning Styles and the Use of ICT in University Students within a Competency-Based Training Model. Journal of New Approaches in Educational Research, 8(1), 1-6. Doi https://doi.org/10.7821/naer.2019.1.296

Consejo de Aseguramiento de la Calidad de la Educación Superior - CACES. (2018). CEAACES. Recuperado de www.caces.gob.ec 
Cai, Yuzhuo. (2017). From an Analytical Framework for Understanding the Innovation Process in Higher Education to an Emerging Research Field of Innovations in Higher Education. The Review of Higher Education, 40(4), 585-616. Recuperado de https://www.researchgate.net/publication/314880078_From_an_Analytical_Framework_f or_Understanding_the_Innovation_Process_in_Higher_Education_to_an_Emerging_Resea rch_Field_of_Innovations_in_Higher_Education

Consejo Superior de Educación - CES. (2018). Página oficial CES. Recuperado de www.ces.gob.ec

Charamba, Tyanai, y Mutasa, Davie. (2018). Folk-story telling among the Shona and Freire 's framework of banking versus dialogical methods of education - In search of innovation and social cohesion in post-independence Zimbabwe 's education. South African Journal of African Languages, 38(2), 189-202. Doi https://doi.org/10.1080/02572117.2018.1463711

Croda, Gabriela., y López, Juan. (2016). The culture of educational innovation in perspective of University managers. Revista de Comunicación Vivat Academia, 134, 58-70. Recuperado de https://search.proquest.com/docview/1782826972?pq-origsite=gscholar

de Anna, Lucia., y Della Volpe, Valentina. (2011). La costruzione di una dimensione internazionale per un e-learning inclusivo. Il Progetto Firb Rete@ccessibile, 10(3), 254266.

de Anna, Lucia., y Covelli, Alessio. (2018). Inclusive Didactics at the University: innovation and training success of students with Special Educational Needs. Form@re, 18(1), 333345. Recuperado

de https://www.researchgate.net/profile/Alessio_Covelli/publication/324603117_Inclusive_Di dactics_at_the_University_innovation_and_training_success_of_students_with_Special_ Educational_Needs/links/5ad88beda6fdcc2935854620/Inclusive-Didactics-at-theUniversity-innovation-and-training-success-of-students-with-Special-EducationalNeeds.pdf

El Comercio. (2018, November 12). La Pro forma registra USD 145,1 millones menos para universidades; Finanzas niega reducción del Fopedeupo. El Comercio. Recuperado de https://www.elcomercio.com/actualidad/ecuador-proforma-universidades-finanzaspresupuesto.html

Ellison, Scott. y Allen, Ben. (2016). Disruptive innovation, labor markets , and Big Valley STEM School: network analysis in STEM education. Cultural Studies of Science Education. Doi https://doi.org/10.1007/s11422-016-9786-9

Evenhouse, David., Patel, Nimit., Gerschutz, María., Stites, Ncki., Roads, Jeffry., Berger, Edward. y Deboer, Jennifer. (2018). Perspectives on pedagogical change : instructor and student experiences of a newly implemented undergraduate engineering dynamics curriculum. European Journal of Engineering Education, 43(5), 664-678. https://doi.org/10.1080/03043797.2017.1397605 
Ferrés, Joan., y Masanet, María-José. (2018). Three paradoxes in the approach to educational technology in the education studies of the Spanish universities. International Journal of Educational Technology in Higher Education, 15. https://doi.org/10.1186/s41239-0180097-y

González-Yebra, Óscar., Aguilar, Manuel., Aguilar, Fernando y Matheu, Manuel. (2018). Evaluación de entornos inmersivos 3d como herramienta de aprendizaje b-learning. Educación XX1, 21(2), 417-440. Doi https://doi.org/10.5944/educXX1.16204

Gros, Begoña. y Lara, Pablo. (2009). Estrategias de innovaciónen la educación superior: el caso de la Universitat Oberta. Revista Iberoamericana de Educación, 49, 223-245. Recuperado de https://rieoei.org/historico/documentos/rie49a09.pdf

Hernández, Elena. y Navarro, María. (2018). Redes educativas locales para la mejora escolar. Revista Electrónica de Investigación Educativa, 20(3), 29-42. Recuperado de https://redie.uabc.mx/redie/article/view/1669/1663

Hod, Yotam., Bielaczyc, Kate., y Ben-zvi, Dani. (2018). Revisiting learning communities: innovations in theory and practice. Instructional Science, 46, 489-506. Doi https://doi.org/10.1007/s11251-018-9467-z

Kim, Leo. y Jang, Deok-Ho. (2017). Expert views on innovation and bureaucratization of science : Semantic network analysis of discourses on scientific governance. Science and Public Policy, 45(1), 1-9.Doi https://doi.org/10.1093/scipol/scx035

Lawrence, Belinda. (2017). Innovating in the real world: exploring institutional effects on tertiary teacher innovations in New Zealand. Distance Education, 39(1), 1-15. https://doi.org/10.1080/01587919.2017.1413932

Le, Hang. (2018). Another textbook project? The implementation of Escuela Nueva in Vietnam. Educational Research for Policy and Practice, 17, 223-239. Doi https://doi.org/10.1007/s10671-018-9230-x

Leat, David. y Thomas, Ulrike. (2018). Exploring the role of ' brokers ' in developing a localised curriculum curriculum. The Curriculum Journal, 29(2). Doi https://doi.org/10.1080/09585176.2018.1445513

Loaiza-Aguirre, María., Andrade-Abarca, Paola. y Salazar-Romero, Ángela. (2017). Determination of the Innovative Capacity of Ecuadorian Universities. Journal of New Approaches in Educational Research, 6(1), 57-63. Doi https://doi.org/10.7821/naer.2016.8.174

Martínez-Usarralde, María., López-Martín, Rámon. y Pérez-Carbonell, Amparo. (2018). EInnovación en la educación superior. Claves para la instutucionalización en las Universidades. Píxel-Bit. Revista de Medios y Educación, 52, 183-197. Recuperado de https://recyt.fecyt.es/index.php/pixel/article/view/62531

Montalvo, Josefa. (2011). Innovación en la educación superior . ¿ Anticipándonos al futuro ? Anuario Jurídico y Económico Escurialense, 567-578. Recuperado de http://www.rcumariacristina.net:8080/ojs/index.php/AJEE/article/view/65 
Murillo-Ribes, Adolf., Riano-Galán, María. y Berbel-Gómez, Noemy. (2018). Percepción sobre el uso de 'Soundcool ' como propuesta de intervención en la creación sonora y en el desarrollo de competencias docentes. Un estudio exploratorio en la formación inicial del profesorado. Psychology, Society, y Education, 10(1), 127-146. Doi https://doi.org/10.25115/psye.v10i1.1051

Niesz, Tricia. y Ryan, Kelly. (2018). Teacher ownership versus scaling up system-wide educational change: the case of Activity Based Learning in South India. Educational Research for Policy and Practice, 17(3), 209-222. Doi https://doi.org/10.1007/s10671018-9232-8

Pinder, Karen. y Shabbits, Jennifer. (2018). Educational leadership during a decade of medical curricular innovation and renewal. Medical Teacher, 40(6), 1-4. Doi https://doi.org/10.1080/0142159X.2018.1440079

Presidencia de la República. (2018). Proyecto de ley Orgánica Reformatoria a la Ley Orgánica de Educación Superior. Recuperado de https://es.slideshare.net/ximenaponceleon/proyecto-de-ley-orgnica-reformatoria-a-la-leyorgnica-de-educacion-superior-tr-183757

Rojo, José. (2007). Regresión lineal múltiple. Recuperado de http://humanidades.cchs.csic.es/cchs/web_UAE/tutoriales/PDF/Regresion_lineal_multiple _3.pdf

Rubio, Francisco. (2012). Innovation and Good Practices in University Government. Revista de Universidad y Sociedad Del Conocimiento, 9(2), 261-266. Recuperado de https://link.springer.com/article/10.7238/rusc.v9i2.1558

Sinclair, Jane. y Aho, Anne. (2017). Experts on super innovators : understanding staff adoption of learning management systems. Higher Education Research y Development, 37(1), 115. Doi https://doi.org/10.1080/07294360.2017.1342609

Scimago Journal Rank - SJR. (2017). SJR. Recuperado de https://www.scimagojr.com/countrysearch.php?country=ec

Solheim, Ksenia., Ertesvåg, Sigrun., y Dalhaug, Grete. (2018). How teachers can improve their classroom interaction with students: New findings from teachers themselves. Journal of Educational Change, 19. Doi https://doi.org/10.1007/s10833-018-9333-4

Teece, David. (2010). Business Models, Business Strategy and Innovation. Long Range Planning, 43, 172-194. Recuperado de http://www.businessmodelcommunity.com/fs/Root/8jig8-

businessmodelsbusinessstrategy.pdf

Thorsteinsson, Gisli. (2013). Ideation training via innovation education to improve students ethical maturation and social responsability. I-Manager's Journal on Educational Psychology, 6(4), 1-8. Recuperado de https://eric.ed.gov/?id=EJ1101766

UNESCO. (2015). International Standard Classification of Education (ISCED) Fields of Education and Training 2013.2 Recuperado de https://doi.org/http://dx.doi.org/10.15220/978-92-9189-179-5-en 
Vélez, Pamela. y Espinosa, Erick. (2018). La planificación financiera y presupuestaria en las instituciones de educación superior de Ecuador. Cofín Habana, 12(2), 280-294. Recuperado de http://scielo.sld.cu/scielo.php?script=sci_arttextypid=S207360612018000200020

Webometrics. (2018). Ranking web de universidades. Recuperado de https://www.webometrics.info/es/WORLD?

Zhou, Guangyou. y Luo, Sumei. (2018). Higher Education Input, Technological Innovation , and Economic Growth in China. Sustainability, 10(2615), 1-15. Doi https://doi.org/10.3390/su10082615 


\section{ANEXO 1: Validación instrumento de encuesta y categorías utilizadas, mediante análisis factorial confirmatorio}

Tabla 9

Categoría 1: Infraestructura, tecnología y recursos

\begin{tabular}{lcccc}
\hline \multicolumn{1}{c}{ Variables } & Comunalidades & $\begin{array}{c}\text { Varianza } \\
\text { explicada }\end{array}$ & Índice KMO & $\begin{array}{c}\text { Alpha de } \\
\text { Crombach }\end{array}$ \\
\hline AU &, 652 & 51,596 &, 871 &, 864 \\
LAB &, 573 & & & \\
BI &, 652 & & & \\
OEC &, 568 & & & \\
EE &, 612 & & \\
PI &, 665 & & \\
CI &, 761 & & & \\
AP &, 703 & & & \\
\hline
\end{tabular}

Elaborado por los autores a partir del estudio "Percepción sobre las necesidades de innovación al interno de las instituciones de educación superior de la provincia de Pichincha, Ecuador".

Tabla 10

Categoría 2: Estructura organizacional

\begin{tabular}{lcccc}
\hline Variables & Comunalidades & $\begin{array}{c}\text { Varianza } \\
\text { explicada }\end{array}$ & Índice KMO & $\begin{array}{c}\text { Alpha de } \\
\text { Crombach }\end{array}$ \\
\hline MDO &, 505 & 47,538 &, 907 &, 841 \\
PC &, 623 & & & \\
PT &, 634 & & & \\
OU &, 557 & & \\
MC &, 533 & & \\
GC &, 662 & & \\
TD &, 627 & & \\
OP &, 608 & & \\
CO &, 562 & & \\
GU &, 525 & & & \\
\hline
\end{tabular}

Elaborado por los autores a partir del estudio "Percepción sobre las necesidades de innovación al interno de las instituciones de educación superior de la provincia de Pichincha, Ecuador". 
Tabla 11

Categoría 3: Personas y competencias profesionales

\begin{tabular}{lcccc}
\hline Variables & Comunalidades & $\begin{array}{c}\text { Varianza } \\
\text { explicada }\end{array}$ & Índice KMO & $\begin{array}{c}\text { Alpha de } \\
\text { Crombach }\end{array}$ \\
\hline RD &, 483 & 56,040 &, 833 &, 801 \\
IN &, 498 & & & \\
PD &, 555 & & & \\
MD &, 624 & & \\
CAS &, 642 & & & \\
\hline
\end{tabular}

Elaborado por los autores a partir del estudio "Percepción sobre las necesidades de innovación al interno de las instituciones de educación superior de la provincia de Pichincha, Ecuador".

Tabla 12

Categoría 3: Plus ofertados por las universidades

\begin{tabular}{|c|c|c|c|c|}
\hline Variables & Comunalidades & $\begin{array}{l}\text { Varianza } \\
\text { explicada }\end{array}$ & Índice KMO & $\begin{array}{l}\text { Alpha de } \\
\text { Crombach }\end{array}$ \\
\hline$\overline{A B M}$ & ,421 & 54,729 & ,845 & ,822 \\
\hline AEX & 583 & & & \\
\hline OFD & ,595 & & & \\
\hline CON & 587, & & & \\
\hline $\mathrm{CCl}$ & 509, & & & \\
\hline OPR & ,589 & & & \\
\hline
\end{tabular}


Revista indizada en

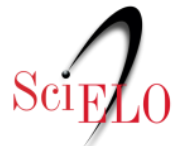

redalyc.sy latindex

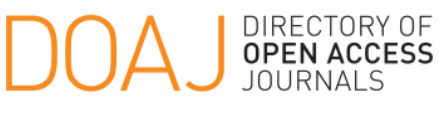

Distribuida en las bases de datos:

- Dialnet

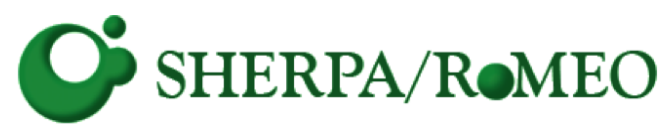

REDIB

Red Iberoamericana

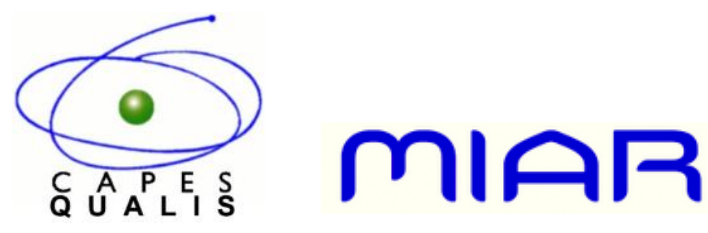

